\title{
Inhaltsverzeichniss des fünf und sechzigsten Bandes.
}

Transformation von Differentialausdrücken erster Ordnung zweiten Grades mit Hülfe der verallgemeinerten elliptischen Coordinaten. Von Herrn O. Henrici zu Kiel. . . . . . . . . . . . . . . . . . . . Seite 1

Beiträge zur Theorie der Variation der einfachen Integrale. Von Herrn

R. Lipschitz zu Bonn. . . . . . . . . . . . . . . . . . . - 26

Ueber die dritte Gattung der Abelschen Integrale erster Ordnung. Von Herrn G. Roch in Halle. . . . . . . . . . . . . . . . . . - 42

Beitrag zur Theorie der ebenen Rouletten. Von Herrn R. Hennig zu Gnesen. - 52 Ueber die Anziehungscomponente eines geraden elliptischen Cylinders in der Richtung der Axe, wenn die Elementaranziehung irgend einer Potenz der Entfernung umgekehrt proportional ist. Von Herrn $F$. Grube zu Hamburg. . . . . . . . . . . . . . . . . . . . . . . 62

Ueber die aus Einheitswurzeln gebildeten complexen Zahlen von peripdischem Verhalten, insbesondere die Bestimmung der Klassenanzahl derselben. Von Herrn L. Fuchs. . . . . . . . . . . . . . . . . . . - 74

Recherche des points à l'infini sur les surfaces algébriques. Première Partie. Par M. L. Painvin à Douai. . . . . . . . . . . . . . . . - 112

Ueber das Verschwinden der $\vartheta$-Functionen. Von Herrn B. Riemann zu Göttingen. . . . . . . . . . . . . . . . . . . . . . . -161

Regel zur Bestimmung des Inhalts der Sternpolygone. Bruchstück aus den hinterlassenen Papieren von C. G. J. Jacobi, mitgetheilt durch Herrn o. Hermes. . . . . . . . . . . . . . . . . . . . . . . - 173

Erläuterung des vorstehenden Jacobischen Bruchstücks. Von Herrn O. Hermes. - 174 Ausdehnung der Jacobischen Regel zur Bestimmung des Inhalts der Sternpolygone für den Fall vielfacher Punkte. Von Herrn O. Hermes. . . - 177

Sur un théorème relatif à huit points situés sur une conique. Par M. A. Cayley a Cambridge. . . . . . . . . . . . . . . . . . . . . -180

Ueber invariantive Elemente einer orthogonalen Substitution, wenn dieselbe als Ausdruck einer Bewegung jeder Gruppe von Werthen der Variabeln aus dem identischen Zustande in den transformirten gefasst wird. Von Herrn Schläfli zu Bern. . . . . . . . . . . . . . . . 185

Programme pour le prix Carpi. . . . . . . . . . . . . . . . . -188 Erweiterung des Satzes, dass zwei polare Dreiecke perspectivisch liegen, auf eine beliebige Zahl von Dimensionen. Von Herrn Schläfli zu Bern. . - 189 
Recherche des points à l'infini sur les surfaces algébriques. Deuxième partie.

Par M. L. Painvin à Douai. . . . . . . . . . . . . . . . Seite 198

Ueber die simultane Integration linearer partieller Differentialgleichungen.

Von Herrn A. Clebsch zu Giessen. . . . . . . . . . . . . . - 257

Ueber diejenigen Curven, deren Coordinaten sich als byperelliptische

Functionen eines Parameters darstellen lassen. Von Herrn Brill. . . - 269

Sur un cas particulier de la surface du quatrième ordre avec seize points

singuliers. Par M. A. Cayley à Cambridge. . . . . . . . . . . - 284

Satz aus der Störungstheorie. Von Herrn Scheibner in Leipzig. . . . . - 291

Ueber einige besondere Punkte des Tetraeders. Von Herrn O. Hermes. . - 293

Sur les sections circulaires des surfaces du second ordre et les ombilics des surfaces quelconques. Par M. C. Souillart à Caen. . . . . . . . - 320

Ueber die Transformation der Abelschen Functionen erster Ordnung. Von Herrn Königsberger zu Greifswald. . . . . . . . . . . . . . - 335

Die Geometrie auf den Flächen dritter Ordnung. Von Herrn A. Clebsch zu Giessen. . . . . . . . . . . . . . . . . . . . . . . . - 359

Ueber die Normalen der Kegelschnitte. Von Herrn C. F. Geiser in Zürich. - 381

Satz aus der Lehre von den Kegelschnitten. Von Herrn O. Hesse in Heidelberg. . . . . . . . . . . . . . . . . . . . . . . . -384

\section{Druckfehlerverzeichniss.}

B a n d 65 .

Pag. 40, Zeile 15 und 16 v. o. Statt des Inhaltes dieser Zeilen bis zum Punkte setze man: $\stackrel{\gamma}{U}_{a}$ folgt unmittelbar, dass $R$ für $x=x_{0}$ bei jedem Werthsystem der Constanten $\stackrel{\alpha, \alpha^{\prime}}{\lambda}$ den Werth der Einheit annimmt.

Pag. 75, Zeile 9. v. o. statt $x$ lese man $x f$.

Pag. 84, Zeile 8. v. u. Im Nenner des Ausdrucks rechts soll der Bruchstrich hinter dem Minus-Zeichen beginnen.

Pag. 97, Zeile 7 v. o. statt „Einheiten der letzteren Art“ lese man „rein imaginäre Einheiten".

Pag. 97, Zeile 8. v. o. statt $\frac{E(\omega)}{E(\omega)^{\zeta}}$ lese man $\frac{E^{\prime}(\omega)}{E(\omega)^{\zeta}}$.

Pag. 97, Zeile 10, 25, 29 v. o. statt $\omega^{\varepsilon}$ lese man $\omega^{-\varepsilon}$.

Pag. 117, Zeile 8 v. o. statt Rouhet lese man Prouhet. 